\title{
XI.
}

(Aus dem Laboratorium der internen Klinik des Prof. A. Gluziński in Lemberg.)

\section{Die sensible und motorische Segmentlocalisation für die wichtigsten Nerven des Plexus brachialis.}

Von

\section{Priv.-Doc. Dr. G. Bikeles und Dr. M. Franke.}

(Mit Tafel III.)

I. Es ist eine vielfach bestätigte Thatsache, dass Extremitätsamputationen nach längerer Zeit hochgradige Schrumpfung, besonders im Hinterstrang der mit der Amputation correspondirenden Seite zur Folge haben. Untersuchungen mit der Marchimethode nach Amputationen, theilweise auch nach Nervendurehschneidung stellten an Darkschewitsch, Langlay und Anderson, Feinberg und Sadowski, dann Redlich (,Die Pathologie der tabischen Hinterstrangserkrankungen" 1897, cit. nach Cassirer). Letzterer fand, dass, wenn die Thiere ganz kurze Zeit gelebt hatten, in den hinteren Wurzeln nach Amputation des Beines keine Degeneration nachweisbar war, nach 36 bis 76 Tagen jedoch fand sich eine solche in den hinteren Wurzeln und dem Hinterstrang der amputirten Seite. Flatau constatirte auch beim Menschen 3 Monate nach der Amputation in dem dem amputirten Gliede entsprechenden Hinterstrang nach Marchi nachweisbare Degenerationen (Deutsche med. Wochenschr. 1897). Cassirer (,Ueber Veränderungen der Spinalganglienzellen und ihrer centralen Fortsätze nach Durchsehneidung der zugehörigen peripheren Nerven". Deutsche Zeitschr. f. Nervenheilk. 1899, Bd. 14) hat bei Kaninchen, bei denen er den N. ischiadicus resecirte, das Rückenmark nach Marchi untersucht und constatirte bei Thieren, die mindestens 20 Tage nach der Resection getödtet wurden, folgende Veränderungen im Lendenmark: Er fand im Hinterstrang der operirten. Seite und zwar dem Verlauf der hinteren Wurzel folgend eine ziemliche Anzahl schwarzer Schollen (Körnchen); diese Degenerationsproducte liessen sich entsprechend den Ausstrahlungen der hinteren Wurzelfasern in die graue Substanz hinein verfolgen. Auch in den vorderen. Wurzeln 
der operirten Seite sah er ebensolche Veränderungen. Dieser positive Erfolg bei Auwendung der Marchimethode hat uns den Gedanken nahe gelegt, ob man nicht auf Grund dieses Befundes die Ursprungsverhältnisse der seusiblen Antheile der Rückenmarksnerven und zwar vorläufig, in der jetzigen Arbeit, die des N. ulnaris, medianus und radialis feststellen könnte. Wenu nämlich nach der Resection eines Nerven in dem intramedullären Theil der hinteren Wurzelfasern, die dem betreffenden Nerven entsprechen, es zu Körnchenablagerung kommt, dann dürfen wir dieses Segment als Ursprungshöhe für die entsprechenden Nervenfasern annehmen, in welchen diese Körnchen in der Wurzeleintrittszone angehäuft sind.

Das Suchen nach einer Methode schien uns geboten, da die Untersuchungen über die Ursprungsverhältnisse der sensiblen Antheile der spinalen Nerven im Allgemeinen wegen Mangels einer bequemen und exacten Methode recht mangelhafte sind. Um Rückschlüsse auf das Verhalten auch beim Menschen machen zn können, haben wir uns vorgenommen, die Ergebnisse bei verschiedenev Thierspecies mit einander zu vergleichen. Darum haben wir unsere Untersuchungen an Kaniuchen, Katzen und Hunden angestellt. ${ }^{1}$ ) Wir resecirten bei je einem dieser Thiere in axilla den $\mathrm{N}$. medianns, ulnaris und radialis zusammen und complet, bei anderen je einen der obengenannten Nerven separat und zwar N. medianus, N. ulnaris oberhalb des Ellbogengelenks, den $\mathrm{N}$. radialis wiederum an der Umbiegungsstelle. Die Thiere wurden 4 Wochen am Leben gelassen. Diese Zeit haben wir auf Grund der Angaben von Cassirer für unsere Wwecke als die beste betrachtet. Cassirer (1. c.) erwähnt nämlich, dass die von ihm beschriebenen Veränderungen erst bej Thieren auftreten, die mindestens 20 Tage nach der Operation getödtet wurden. Am ausgesprochensten waren sie dagegen bei denen, die 30 Tage die Operation überlebten, später nahmen sie an Intensität schon sehr ab und sind nach 63 Tagen eben nur noch erkenmbar.

Zur anatomischen Untersuchung wurden immer vor Allem dünne Stücke des Rückenmarks aus dem Niveau der Wurzelinsertion entnommen und wie gewöhnlich nach Marchi behandelt. Was den intramedullären Abschnitt der Hinterwurzelfasern betrifft, konnten wir bei Kaninchen, Katzen und Hunden das Vorhandensein deutlicher Veränderungen in denselben auf der Seite der ausgeführten Nervenresection constatiren. Wir fanden nämlich, dem Wurzelfaserverlaufe im Rücken-

1) Bei Meerschweinchen haben wir ein negatives Resultat bekommen, vielleicht war die Zeit, die seit der Resection verflossen, zu kurz. Darkschewitsch (Neurol. Cbl. 1892) sah bei Meerschweinchen 6 Wochen nach der Resection eine grosse Anzahl schwarzer Schollen im Hinterstrang der operirten Seite. 
Sensible u. motorische Segmentlocalisation f. d. wichtigsten Nerven ete. 207

marke entsprechend, Ablagerung zahlreicher schwarzer Körnchen (Schollen) zerfallenen Myelins, und zwar waren diese Körnchen in einer grösseren oder geringeren Anzahl der unteren Segmente in der Wurzeleintrittszone angehäuft, weiter oben dagegen rückte die ganze Körnchenmasse immer mehr und mehr gegen die Mittellinie, dabei auch immer mehr an Zahl sich verringernd (vgl. Taf. III A), also ganz ähnlich dem bekannten Bilde der secundären Degeneration nach Läsion der hinteren Wurzeln selbst. Und wir haben dieses Segment, in welchem diese schwarzen Körnchen in der Wurzeleintrittszone, sei es ausschliesslich, sei es in Gemeinschaft mit der Mittelzone, auftreten, als Ursprungssegment des resecirten Nerven betrachtet.

Dass Knape (,Ueber Veränderungen im Rückenmark nach Resection einiger spinaler Nerven u.s. w." Beiträge zur pathol. Anatomie 1901) beim Kaninchen nach Resection eines oder sogar zweier Nerven am Cubitalgelenk mit Marchimethode keine Veränderungen im Rückenmark constatiren konnte, kann nur mit einem Uebersehenwerden von beim Kaninchen nach Medianus- und Ulnaris-Resection allerdings minimalen Degenerationen erklärt werden.

Wenn wir zu unseren Einzelbefunden bezüglich der Ursprungsverhältnisse der einzelnen Nerven übergehen, so baben wir Folgendes:

1. Bei je einem Kaninchen, Hund und Katze wurden in axilla der N. radialis, medianus und ulnaris zusammen complet resecirt, und wir fanden zahlreiche feine, hart bei einander angehäufte schwarze Schollen in der Wurzeleintrittszone des Hinterstranges auf der operirten Seite sowohl beim Kaninchen (vgl. Taf. 1II A) als beim Hunde und bei der Katze, reichlich auf der Höhe des siebenten und achten Cervical-, relativ gering, doch sehr deutlich erkennbar auf der Höhe des ersten Dorsalsegmentes. Auf der Höhe des sechsten Cervicalsegmentes ist beim Kaninchen die Wurzeleintrittszone gänzlich von den Schollen frei, wäbrend bei der Katze und beim Hund die Hauptmasse derselben allerdings diese Zone bereits verlassen hat, doch erscheint sie von denselben nicht ganz frei.

Wir können somit annehmen, dass bei allen drei Thierspecies das Gros der sensiblen Fasern der drei Hauptnerven für die vordere Extremität mit der siebenten wie achten Cervical- und ersten Dorsalwurzel in das Rückenmark eintritt, bei Katze und Hund mag auch , ein winziger Theil der Fasern in der sechsten hinteren Cervicalwurzel verlaufeu. Ob nicht einzelne wenige Fasern oberhalb dieser Segmente ihren Weg nehmen, möchten wir nicht ausschliessen, da einzelne Fasern, peripher vom Spinalganglion durchschnitten, keine oder fast keine Veränderungen hervorrufen können.

Nach Resection eines einzelnen Nerven des Plexus waren im All- 
gemeinen die Veränderungen quantitativ viel geringer, besonders beim Kaninchen, aber immer deutlich genug, um sie selbst beim Kaninchen als solche zu erkennen.

2. Nach Resection des Nerrus medianus oberhalb des Ellbogengelenkes haben wir in der Wurzeleintrittszone fast derselben Segmente wie bei 1., d. i. im siebenten und achten Cervical-, dann im ersten Dorsalsegment, nur quantitativ viel geringere Veränderungen constatirt (vgl. Taf. III C). Bein Hund und bei der Katze waren diese Veränderungen im siebenten und achten Cervicalsegment sehr deutlich entwickelt, beim Kaninchen dagegen überhaupt eben noch kaum zu erkennen. Das Gros der sensiblen Antheile des Nervus medianus tritt also bei allen drei Thierspecies durch die siebente und achte und nur wenige Fasern durch die erste Dorsalwurzel in das Rückenmark ein.

3. Wenn wir den N. ulnaris resecirten, dann fanden wir beim Hund und bei der Katze (vgl. Taf. III D) die Körnchenablagerung sehr dentlich in der $W$ urzeleintrittszone des achten Cervical- und weniger deutlich in der des ersten Dorsalsegments. Beim Kaninchen bestanden dieselben Verhältnisse, aber die Körnchenablagerung war bei letzterem überhaupt sehr gering, eben noch kaum zu constatiren. Für den N. ulnaris kommt also in Betracht nur das achte Cervicalund erste Dorsalsegment; die meisten Fasern entspringen aus der Höhe des achten Cervicalsegmentes.

4. Den N. radialis resecirten wir separat nicht in axilla, sondern an der Umbiegungsstelle, wo er aus der Tiefe herauskommt, so dass der Ast für den Triceps erhalten blieb. In diesem Falle war auch beim Kaninchen (vgl. Taf. III B) eine grosse Anzahl von schwarzen Schollen - natürlich quantitativ viel geringer als nach completer Resection aller 3 Nerven - in der Wurzeleintrittszone auf der Höhe des sjebenten und achten Cervical- und nur eine geringe in der Höhe des ersten Dorsalsegmentes anzutreffon. Bei der Katze war der Befund im ersten Dorsalsegment negativ, im siebenten und achten Cervicalsegment dem beim Kaninchen gleich. Leider ist das Material vom Hund durch irgend eine Verunreinigung der Marchi'schen Flüssigkeit unbrauchbar geworden. Obwohl nun die separate Resection des $\mathrm{N}$. radialis nicht an der Abgangsstelle desselben in axilla ausgeführt wurde, können wir doch diese Resection als ziewlich ausreichend betrachten, da der Radialis oberhalb der von uns gewählten Stelle keine grösseren sensiblen Aeste abgiebt, und besonders da die Veränderungen in dem Gebiete der Wurzeleintrittszone nach Resection aller 3 Nerven nicht wesentlich weiter hinaufreichte. Wir finden also beim Kaninchen als Eintrittshöhe das siebente und achte Cervical- und erste Dorsalsegment, bei der Katze nur das siebente und achte Cervical- 
Sensible u. motorische Segmentlocalisation f. d. wichtigsten Nerven etc. 209

segment. Beim Kaninchen treten auf der Höhe des sechsten Cervicalsegments sicher keine Fasern ein, bei der Katze dagegen, bei der wir nach Resection aller 3 Nerven in axilla im sechsten Cervicalsegment eine geringe Ablagerung von Körnchen in der Wurzeleintrittszone angetroffen haben, würde für den Radialis auch das sechste Cervicalsegment in geringem Grade in Betracht zu ziehen sein.

Wenn wir das gesammte Resultat zusammenfassen, so sehen wir, dass die sensiblen Antheile des N. radialis, medianus und ulnaris aus mehreren Segmenten, wie allgemein auch angenommen wird, entspringen und zwar a) der $\mathrm{N}$. radialis aus dem siebenten und achten Cervical-, beim Kaninchen anch aus dem ersten Dorsalsegment, bei der Katze und wahrscheinlich ebenso beim Hund in geringer Menge auch aus dem sechsten Cervicalsegment, b) der N. medianus ans der Höhe des siebenten und achten Cervical- und ersten Dorsalsegmentes und c) der N. ulnaris nur aus dem achten Cervical- und ersten Dorsalsegment.

In die Augen springend ist die bei allen von uns untersuchten Thieren festgestellte ziemliche Harmonie in der Ursprungshöhe der sensiblen Antheile der erwähnten Nerven und lässt sich nur hier und da eine kleine Abweichung constatiren. Diese Harmonie berechtigt zur Annahme, dass die Ursprungsverhältnisse bei anderen Thierspecies denen von uns untersuchten gleichen dürften.

Was speciell das Verhalten beim Menschen anbetrifft, so differiren die Angaben der Forscher in ganz erheblichem Grade (vgl. die Zusammenstellungen bei Wichmann, „Die Rückemarksnerven und ihre Segmentbezüge". 1900, S. 88, 92, 102). Es ist dies um so weniger befremdend, als Wichmann (l. c. S. 199) auch die Schemata über die Rückenmarkssegmentation am Arm von Allen Starr, Thorburn und Kocher "alle verschieden" findet und den Mangel einer Uebereinstimmung zwischen denselben mit den von Head bei Zoster gewonnenen Resultaten hervorhebt. Am nächsten unseren Ergebnissen kommen die von Wichmann (l. e.) im zweiten klinisehen Theil kritisch corrigirten Daten betreffs der Rückenmarkssegment-Innervation des Hautgebietes des Cerv. 5 - Thor. I. Daraus (S. 200-205) würde resultiren, dass beim Menschen für den Radialis das sechste und siebente, für den Medianus das siebente und achte, endlich für den Ulnaris das achte Cervical- und erste Dorsalsegment in Betracht kommen. Es ist aber für uns wahrscheinlich, dass in Analogie mit unseren Ergebnissen bei Thieren auch beim Menschen für den Radialis noch das achte Cervical-, für den Medianus das erste Dorsalsegment in Anspruch zu nehmen ist. Man muss nämlich bedenken, dass gerade beim $\mathrm{Zu}$ grundelegen von klinischen Beobachtungen die Betheiligung des untersten Segmentes, falls es sich um eine Innervationsüberlagerung 
dreier Segmente wie am Vorderarm und Hand handelt, leicht übersehen werden kann. Denn mit Ausnahme ganz seltener Fälle von Wurzelläsionen betreffen die einschlägigen klinischen Beobachtungen Querschnittserweichungen im Rückenmark, meist in Folge von Trauma. Da nun wohl bei Wurzelaffectionen, keineswegs aber bei Querschnittserkrankungen ein isolirtes Erhaltensein der Sensibilität seitens des untersten, an der Hautinnervation einer bestimmten Region betheiligten Segmentes bei Zerstörung des entsprechenden oberen Segmentes möglich ist, so ist auf Grund klinischer Forschung das Antreffen von erhaltener Sensibiltät in Folge isolirter Intactheit des tieferen Segmentes ungemein erschwert. Andererseits können wiederum erhaltene zwei höhere Segmente so sehr für die Herstellung der Sensibilitït einer bestimmten Region ausreichen, dass der Ausfall von, auch relativ reichlichen, Fasern aus einem tieferen Segment gänzlich entgehen mag. Leider sind anch die wenigen mitgetheilten klinischen Fälle von Wurzelläsion (Pfeiffer, Deutsche Zeitschr. f. Nervenh. I и. J. Müller, Deutsche Zeitschr. f. Nervenh. V) nicht stricte beweisend.

Die gate Uebereinstimmung zwischen dem Verhalten beim Thier und Menschen, zugleich auch die Verlässlichkeit nnd Sicherheit der von uns angewendeten Methode beweisen die Resultate von nachträglich ausgeführten hohen Plexusresectionen, d. i. vach Resection des Plexus brachialis oberhalb der Clavicula. Diese Operation wurde an je einem Kaninchen und Hund ausgeführt und die Ergebnisse waren folgende: Beim Kaninchen, wo die Obduction eine complete Plexusresection bestätigte, erstrecken sich die Veränderungen in der Wurzeleintrittszone der operirten Seite vom fünften Cervical- bis inclusive ins zweite Dorsalsegment, in letzterem allerdings nur minimal. Beim Hund, bei welchem die Obduction das Erhaltensein des untersten, offenbar den untersten Segmenten entsprechenden Astes ergab, beginnen ebenfalls die Veränderungen in der Wurzeleintrittszone des fünften Cervicalsegments, werden zahlreicher auf der Höhe des sechsten und siebenten, verringern sich aber schon beträchtlich im achten Cervicalsegment und erscheinen nur noch spurweise im ersten Dorsalsegment. Der constatirte Beginn der seusiblen Fasern des Plexus brachialis bei Kaninchen und Hund im fünften Cervicalsegment stimmt nun ganz überein mit den klinischen Beobachtungen beim Menschen nach Totalquerläsion des fünften Cervicalsegmentes (vgl. Kocher, Mittheilungen aus dem Grenzgebiet ete. I, auch Wichmann l. c.).

Im Früheren befassten wir uns blos mit den Veränderungen im intramedullären Theile der Hinterwurzelfasern. Die Autoren beschreiben nun auch analoge Veränderungen im intramedullären Verlauf der vorderen Wurzeln. Thatsächlich haben wir beim Hunde 
Ablagerung von schwarzen Körnchen längs des intramedullären Verlaufes der vorderen Wurzeln tief in das Vorderhorn hinein constatiren können. Die Körner sind hier schön reihenweise angeordnet und ziehen im Vorderhorn der operirten Seite deutlich in der Richtung nach den lateralen Ganglienzellengruppen hin. In der Richtung nach der medialen Gruppe hingegen sahen wir dieselben sich niemals erstrecken, und es war letztere Gegend, was gleich hervorgehoben werden soll, immer von Köruchen (Schollen) frei. Dieses Auftreten von Schollen längs des intramedullären Verlaufes der Vorderwurzelfasern erfolgt nach Resection der N. radialis, medianus und ulnaris zusammen in geringem Grade schon im siebenten Cervicalsegment, am deutlichsten und schönsten im achten Cervical-, dann im ersten Dorsalsegment (im letzteren weniger deutlich als im achten Cervical-, aber stärker als im siebenten Cervicalsegment). Nach Resection des Medianus wie nach der des Ulnaris beim Hund war diese Veränderung nur im achten Cervicalsegment vorhanden. Nach hoher Resection des Plexus brachialis, bei der aber der unterste Ast erhalten geblieben ist, finden sich im intramedullären Verlaufe der vorderen Wurzeln reihenweise angeordnete Ablagerungen von schwarzen Körnchen (Schollen) im sechsten und siebenten Cervicalsegment, und auch hier vermisst man jedes Hinziehen der Schollen in der Richtung zu den medialen Gruppen. Bei der Katze und beim Kaninchen waren die Körnchen an den Vorderwurzelfasern vicht deutlich vorhanden.

Bezüglich der Natur der im Obigen beschriebenen, bei Marchifärbung nachweisbaren Veränderungen im Hinterstrang nach Nervenresectionen führt Cassirer nach Redlich drei Möglichkeiten an: 1. degeneriren vielleicht nur solche Fasern, die das Spinalganglion blos passiren, ohne Unterbrechung zu erfahren, oder 2. die Degeneration könnte Folge einer Veränderung der Spinalganglienzellen sein, oder 3. endlich handelt es sich um den Effect eines Traumas (Zug, Zerrung am Spinalganglion und der hinteren Wurzel). Die letztere Möglichkeit, dass diese Veränderung blos zufällig durch eine Complicirtheit mit einer starken Einwirkung des Traumas bedingt sein könnte, müssen wir in Uebereinstimmung mit Cassirer selbst, entgegen der Meinung Knape's (1. c.) entschieden zurückweisen, da die Veränderung auch bei schonendster Ausführung der Operation in beträchtlicher Entfernung vom Rückenmarke bei bester Wundheilung erfolgte.

Die erste Annahme wiederum, dass es sich um Fasern handle, die aus an der Peripherie gelegenen Ganglienzellen entspringen (Pierre Marie, Lecons sur les mal. etc. 1892), müssen wir auf Grund unserer Untersuchungen (Przegląd lekarski 1902) als unannehmbar erklären. Auch sind die bei unseren Untersuchungen angetroffenen schwarzen Schollen 
sehr zart und fein, ganz anders wie bei der gewöhnlichen Waller'schen Degeneration. Es handelt sich auch da nicht um den Zerfall der Markscheide einer Nervenfaser in ihrer Totalität wie bei der Waller'schen Degeneration, sondern um langsam sich abspielende die Nervenfaser nur theilweise schädigende atrophische Vorgänge, die nach Nervenresection ebenso wie nach Amputation in erster Linie die Markscheide betreffen. Zur Erklärung des Auftretens solcher Veränderungen in den Wurzelfasern oberhalb des Spinalganglions hat allerdings die Annahme Cassirer's, wonach die Veränderungen der Hinterwurzelfasern Folge von vorausgegangenen Alterationen in den Spinalganglienzellen sein sollten, viel Bestechendes an sich. Doch stricte bewiesen ist dies nicht, und könnten vielleicht deunoch die Veränderungen in Spinalganglienzellen und Hinterwurzelfusern trotz des beträchtlichen frühzeitigen Sichtbarwerdens derselben in ersteren coordinirte Erscheinungen, bedingt durch die Läsion des peripheren Fortsatzes derselben Zelle, sein. Dafür scheint auch der Umstand zu sprechen, dass lange Jahre nach stattgehabter Amputation die Veränderungen im Hinterstrange gerade enorme sind, keineswegs aber in den entsprechenden Spinalganglienzellen.

II. Während wir behufs Constatirung der Ursprungshöhe der sensiblen Antheile der im Vorigen angeführten Nerven vom Rückenmarksegment die Hauptinsertionsstelle der Wurzeln zur Färbung wach Marchi verwendeten, benutzten wir dünnere oder dickere Stücke von ober- und unterhalb ersterer Stelle zur Behandlung nach Nissl, um auch über die Herkunft der motorischen Fasern Aufklärung zu erhalten. Die daraus gewonnenen Schnitte wurden meist mit Thionin, oft aber auch mit Neutralroth gefärbt. Die Veränderungen, die wir dann antrafen, sind die unter der Bezeichnung Degeneratio axonalis hinreichend bekannten, also ausgesprochene totale oder seltener auch partielle Chromatolyse, recht deutliche excentrische, oft hernienartige Verlagerung des Kerns, häufig Anschwellung des Zellleibes. Besonders ausgeprägt war diese Nissl'sche Degeneration bei Hund und Katze, bei weitem weniger bei Kaninchen und Meerschweinchen, weshalb auch von uns besonders das Verhalten bei ersteren eine genauere Berücksichtigung fand. Voransgeschickt mag hier gleich werden, dass wir niemals im Vorderhorn der zweiten nicht operirten Seite auch nur eine einzige unzweifelhaft pathologische, d. i. die früher erwähnten Veränderungen zeigende Zelle sehen konnten.

Speciell zu den Befunden beim Hund übergehend, so verfügen wir über die Ergebnisse aus je einem Fall nach Resection 1. des N. radialis (Umbiegungsstelle), 2. einer solchen des N. medianus, 3. dann des N. ulnaris und endlich 4. aller 3 Nerven zusammen (in letzterem Falle 
auch Tricepsast durchschnitten). Die auf diese Weise gewonnenen Resultate stellen sich in Kürze folgendermassen dar:

a) Bezüglich der Höhenlocalisation fanden wir sowohl nach completer Resection aller 3 Nerven als auch nach einer solchen des alleinigen $\mathrm{N}$. radialis an der Umbiegungsstelle unmittelbar unterhalb der Insertionsstelle der siebenten Cervicalwurzel ausgesprochene Bilder axonaler Degeneration beim gäuzlichen Fehlen derselben oberhalb der siebenten Cervicalwurzel. Die Insertionsstelle selbst verbrauchten wir anderweitig zur Marchifärbung, werden aber kaum fehlgehen in der Annahme, dass gerade in der Austrittshöhe der siebenten motorischen Wurzel der Anfang für das reichliche Auftreten von motorischen Zellen des N. radialis zu suchen ist. Nach Resection, sei es des N. medianus, sei es des N. ulnaris, zeigten die Schnitte aus dem siebenten Cervicalsegment (d. i. von ober- und unterhalb der Wurzelinsertionsstelle) keinerlei pathologische Veränderungen, und traten solche erst unmittelbar oberhalb der Insertionsstelle der achten Cervicalwurzel auf, sind aber auch daselbst geringer als unmittelbar unterhalb der Austrittshöhe der achten motorischen Wurzel. Besonders nach Resection des N. ulnaris ist die Anzahl der axonale Degeneration zeigenden Vorderhornzellen oberhalb der Insertionsstelle der achten Cervicalwurzel gering. In der Richtung nach abwärts fanden sich noch nach Resection je eines der 3 in Rede stehenden Nerven Zellveränderungen unmittelbar oberhalb des Austrittes der ersten Dorsalwurzel, fast gar nicht dagegen unmittelbar unterhalb. Auf Grund dessen dürften wir annehmen, dass beim Hunde die motorisehen Fasern

1. des N. radialis rom siebenten, achten Cervicalis und ersten Dorsalis,

2. des N. medianus vom achten Cervicalis und ersten Dorsalis,

3. des $\mathrm{N}$. ulnaris vom achten Cervicalis

(im oberen Theil weniger reichlich als Medianus) und ersten Dorsalis hauptsächlich abstammen. Diese unsere Resultate betreffs der motorischen Segmentlocalisation stimmen ziemlich gut überein mit denen Marinesco's (Contribution a l'étude des localisations des noyaux moteurs dans la moelle épinière. Rev. neurol. 1898, dann „Localisations motrices spinales" Rev. neurol. 1901), welcher ebenfalls den Radialis hauptsächlich rom siebenten Cervicalis (nur minimal vom sechsten, „wenige zerstrente Zellen") an entspringen lässt und für den Ursprung des Medianus erst das untere Drittel des siebenten Cervicalis („viel höher" als für den Ulnaris) in Anspruch nimmt. Die Angaben Knape's (l. c.), besonders über die Ausdehnung "des Ulnariskernes" im siebenten, achten Cervicalis und ersten Dorsalis sind entschieden zu weit gehend und dürften dieselben damit erklärt werden, dass dieser Autor auch 
nicht ganz unzweifelhafte pathologische Veränderungen verwerthete (vgl. 1. c. S. 275).

b) Ausser der Segmentlocalisation ist aber auch die Frage nach der Abstammung von speciellen Zellgruppen des Vorderhornes ron Wichtigkeit. Die distinctesten Zellgruppen dieser Region sind die ventro-mediale, die ventro-laterale, die dorso-laterale, dann die centrale; aber schon deren Form und Zellenreichthum wechselt, wie schon Marinesco zugesteht, von Schnitt zu Schnitt innerhalb desselben Segmentes. Zwischen den Hauptgruppen befinden sich nun Zellhaufen, die stellenweise ziemlich amgrenzt, bald wiederum doch nur einen diffusen Uebergang ersterer darstellen, so dass weitere specielle Gruppenbenennungen oft mehr schematischen Zwecken als zum Ausdruck wirklicher morphologischer Besonderheiten dienen. Eine solche Bezeichnung ist "die intermediäre" (Marinesco) für die zwischen dorso-lateraler und ventro-lateraler Gruppe gelegenen Zellen, welche für uns von Interesse sind.

In allen unseren Fällen fanden wir beim Hund die ventromediale wie die centrale Gruppe, dann den vordersten Theil der lateralen ausnahmslos von Nissl'scher Degeneration frei. Die Veränderungen nach Resection, sei es des Medianus, sei es des Ulnaris oder des Radialis an der Umbiegungsstelle, beschränkten sich stets einzig anf die dorsolaterale Gruppe, welche dann jedesmal eine gewisse Anzahl von alterirten Zellen enthielt. Nach Resection des N. medianus oder ulnaris befinden sich die pathologischen Zellen im medialen Theil der dorsolateralen Gruppe, wo sie allerdings bis an den hinteren Vorderhornrand beraureichen; nach Resection des Radialis (Umbiegungsstelle) hingegen befinden sich die degenerirten Zellen ganz im lateralen Abscbnitt dieser Gruppe. In dem Falle, in welchem ausser dem Ulnaris und Medianus auch noch der Radialis complet (sammt dem Tricepsast) resecirt wurde, betrifft die axonale Degeneration schon im siebenten Cervicalis fast die ganze dorso-laterale Gruppe und im achten Cervicalis oceupirt dieselbe auch noch die sogenannte intermediäre Gruppe, so das im letzteren Segment von den lateralen Zellgruppen nur die vordersten, fast ventral gelegenen Zellen gut erhalten sind.

Man ersieht daraus, dass der Triceps (Anconeus-)ast des Radialis für sich im siebenten Cervicalis den grössten Theil der dorso-lateralen, im achten Cervicalis die ,intermediäre" Gruppe in Anspruch nimmt, was wohl mit den Ergebnissen Marinesco's (l. c.) und seiner Schüler (Parhon und Goldstein, Neurol. Centralbl. 1901) tubereinstimmt. Den übrigen Aesten des Radialis entsprechen ausschliesslich in der dorsolateralen Gruppe lateral und meist im siebenten und achten Cervicalsergment nach hinten gelegene Zellen, während die Nn. medianus und 
ulnaris überwiegend gerade aus dem medialen Theil dieser Gruppe entspringen, und können wir auch in dieser Beziehung die Angaben Marinesco's und seiner Schüler (1. c.) bestätigen; auffallender Weise gelangt Kn a p (1.c.) zu einem ganz entgegengesetzten Resultate (Flexorengruppen lateral, Extensorengruppen mehr in der Mitte des Hornes).

Bei der Katze differiren einigermassen unsere Befunde bezüglich der Höhenlocalisation von den beim Hund von uns notirten, doch bleibt das gegenseitige Verhältniss für den Ursprung der motorischen Antheile der 3 Nerven unverändert. Es ergeben sich nämlich:

für den Radialis sechster (wenig unterhalb), siebenter, achter Cervicalis und erster Dorsalis,

für den Medianus siebenter (unterhalb), achter Cervicalis und erster Dorsalis,

für den Ulnaris siebenter (unterhalb), achter Cervicalis und erster Dorsalis.

Auch bei der Katze ist die axonale Degeneration nur in den lateralen Zellengruppen des Vorderhornes anzutreffen. Auf sonstige Einzelheiten soll hier nicht eingegangen werden, da dieselben noch einer Nachuntersuchung benöthigen. Es sei nur bemerkt, dass auch bei der Katze die Ursprungszellen für den Medianus und Unaris innerhalb der lateralen Gruppe überwiegend medial gelagert sind, während die des Radialis vorwaltend im lateralen Abschnitt sich finden.

Zum Schlusse wollen wir noch unsere Ergebnisse beim Kaninchen und Meerschweinchen nach completer Resection aller drei Nerven (Radialis, Ulnaris, Medianus) zusammen erwähnen. Beim Kaninchen betraf dann die Nissl'sche Degeneration das sechste (wenig), siebente, achte Cervical- und (nur minimal oberhalb der Wurzelinsertion) das erste Dorsalsegment. Die reichlichste Anzahl alterirter Zellen fand sich unmittelbar oberhalb des Austrittes der achten Cervicalwurzel. Beim Meerschweinchen constatirten wir die Veränderungen im sechsten (wenig), siebenten und achten Cervicalsegment. Sitz der Nissl'schen Degeneration waren auch bei diesen beiden 'Thierspecies die lateralen Vorderhorngruppen.

\section{Erklärung der Tafel III.}

Dieselbe zeigt die Segmentausdehnung der innerhalb der Wurzeleintritts. zone des Hinterstranges constatirten Veränderungen und zwar:

A) beim Kaninchen nach completer Resection aller 3 Nerven (Radialis, Medianus und UInaris); daselbst auch die höher oben erfolgende Verschiebung der Schollen gegen das Sept. med. post, sichtbar.

B) ebentalls beim Kaninchen nach Radialis-Resection(Umbiegungsstelle).

O) beim Hund nach Medianus-Resection.

D) bei der Katze nach Ulnaris-Resection. 
Deutsche Zeitschrift E.Nervenheilkunde Bd.XXIII.

Tafel III.

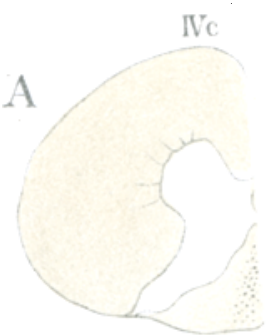

VII

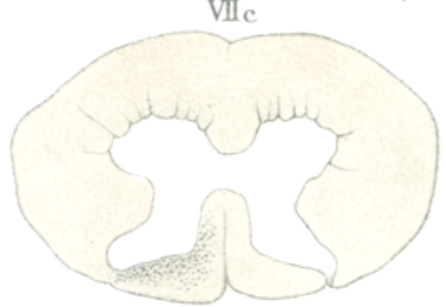

VIIIC

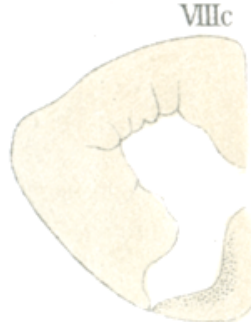

VIc
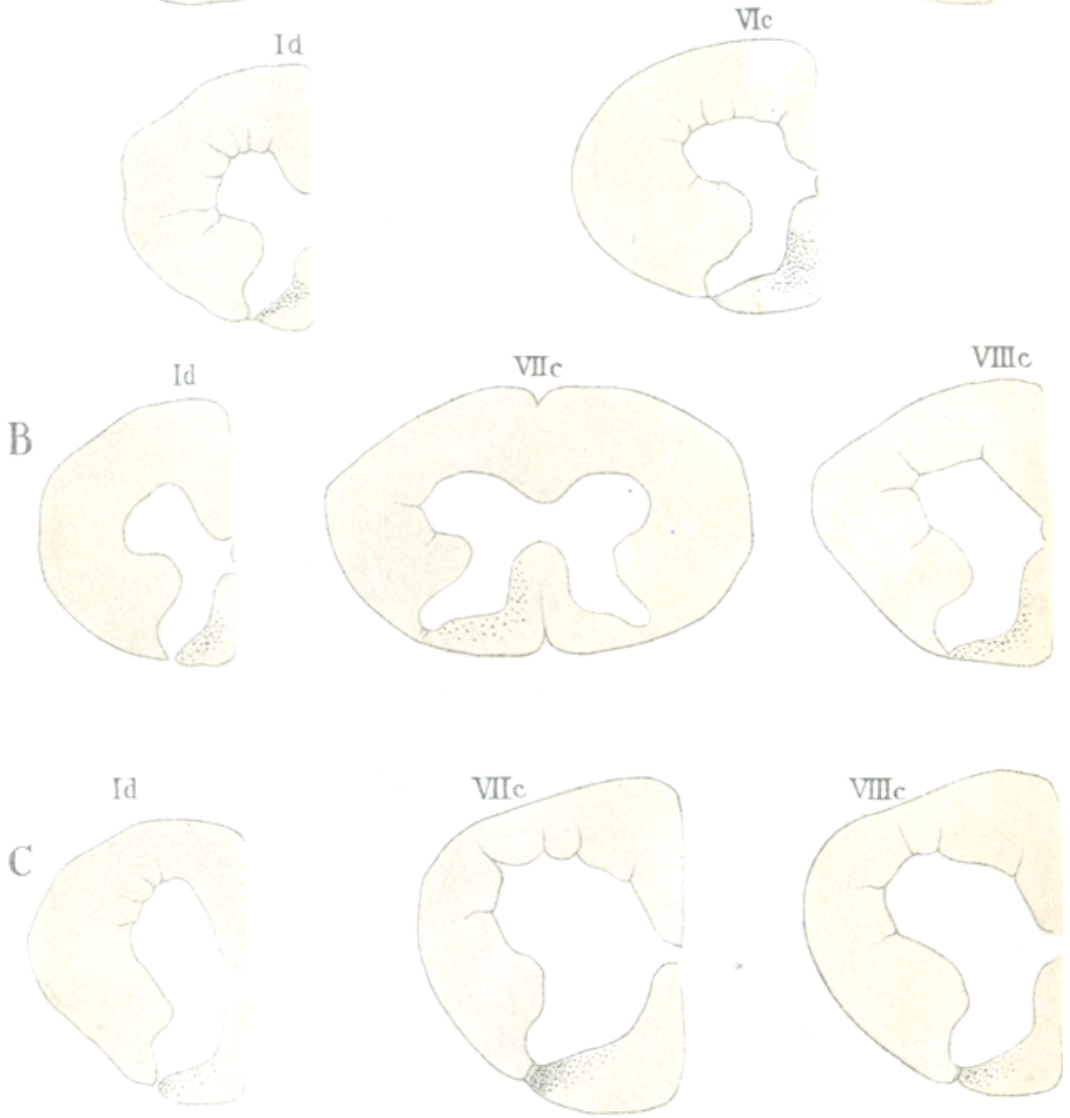

Id

D)

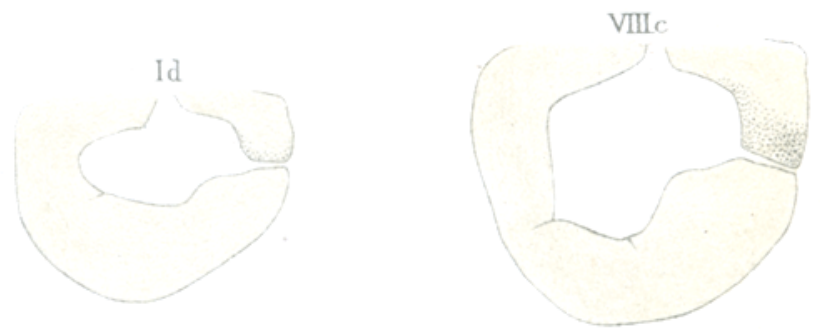

Bikelesuftake

F.C.Woges

Hathen 